\title{
Globally Stable Fast Tracking Control of A Chain of Integrators With Input Saturation and Disturbances: A Holistic Approach
}

\author{
$\mathrm{Lu} \mathrm{Lu}$ and Bin Yao
}

\begin{abstract}
A new approach to the globally stable tracking control of multiple integrators with input saturation and bounded disturbances is proposed. The controller has a hybrid structure. Specifically, in the inner loop, a nonlinear control law is designed in continuous-time domain to have an arbitrarily good disturbance rejection performance at the steady-state while keeping the tracking errors with respect to on-line replanned trajectory within certain positive invariant set even in the presence of input saturation and bounded disturbances, provided that the replanned trajectory satisfies certain conditions. In the outer loop, a trajectory replanning unit implemented in discrete-time domain is constructed to generate a replanned trajectory satisfying those conditions while minimizing the converging time of the replanned trajectory to the desired target. It is theoretically shown that the resulting closed-loop system is globally stable and can track any feasible desired trajectory with a guaranteed steady-state tracking accuracy. Comparative simulation results have been obtained to verify the superior performance of the proposed controller over various existing ones in terms of the disturbance rejection capability and the overall respond speed of the resulting closed-loop system for a third-order integrator chain.
\end{abstract}

Index Terms - Integrators; Saturation; Trajectory Planning; Nonlinear Control

\section{INTRODUCTION}

Any meaningful control design needs to take various implementation constraints into account. Input saturation, due to its universal presence in physical systems caused by the limited actuator power, has been used as a typical example of practical constraints. At the same time, to justify the additional cost needed to implement feedback controllers in any industrial application, the control design needs to focus more on the improvement of overall system performance (e.g., faster responses and better disturbance attenuation capability) rather than the closed-loop stability only. It is well known that, even for systems described by multiple integrators, controllers from traditional linear feedback control designs could easily become destabilizing when the control input is saturated [3]. As such, in spite of the seemingly simple dynamics of multiple integrators, they have been used as a benchmark example in the control of systems with input saturations [14], [11], [19], [16], [8], [18], [10].

The work is supported in part by the US National Science Foundation (Grant No. CMS-0600516) and in part by the National Natural Science Foundation of China (NSFC) under the Joint Research Fund for Overseas Chinese Young Scholars (Grant No. 50528505) and the Ministry of Education of China through a Chang Jiang Chair Professorship.

$\mathrm{Lu} \mathrm{Lu}$ is currently a graduate student of Mechanical Engineering, Purdue University, West Lafayette (lu27@purdue.edu)

Bin Yao is a Professor of School of Mechanical Engineering at Purdue University, West Lafayette, IN 47907, USA (byao@purdue.edu).
The global stabilization of a chain of integrators with input saturation was first solved in [19] in which a state coordinate transformation approach was proposed. Since then, a large number of publications appeared along the same line of thought process in dealing with input saturation [16], [8]. As pointed out in [8], [10], this class of algorithms have the significant drawback that it can not deal with large input disturbances. The input saturation level has to be greater than $2^{n}-1$ times the level of disturbance to ensure stability, where $n$ is the order of the system. Other feedback approaches that explicitly deal with the input saturation problem exist as well such as the anti-windup techniques [18], [9] and the low-and-high gain approaches [14], [11]. Theses designs focus on how to enlarge the domain of attraction by properly designing the linear feedback control laws while meeting certain performance requirements such as disturbance rejection. However, the converging times to the desired trajectory are not guaranteed to be globally optimal because the design is purely based on the scheduling of feedback gains.

Besides the above approaches which specifically deal with systems with input saturation, the Model Predictive Control (MPC), which has been gaining popularity in recent years [13], [4], [12], can also be used to solve the input saturation problem while minimizing the converging time due to the powerfulness of MPC in dealing with hard constraints and its optimization capability. However, due to the computational complexity of MPC, the controller has to be implemented with slow sampling rates. This drawback significantly limits the usefulness of MPC when high sampling rates are required to obtain good disturbance rejection capability or to achieve excellent tracking performance in the presence of model uncertainties.

As an alternative to MPC in dealing with hard constraints, a reference governor (RG) approach has also been developed [1], [6], [15], [7]. RG assumes that a low level stabilizing controller has already been designed in the inner loop with good disturbance rejection capabilities when no hard constraints such as the input saturation occur. A reference governor using certain constrained optimization algorithms is then constructed at a higher level to regulate the reference command sent to the inner stabilizing controller in order to prevent constraint violations. However, no efforts have been made to investigate the interaction between the inner stabilizing controller and the outer reference governor. Thus, RG is difficult to achieve both fast transient response speed and good disturbance rejection at steady state simultaneously as will be seen in the simulation section.

Having known the merits and limitations of traditional 
approaches, a novel hybrid control structure shown in Fig 1 is proposed to solve the problem in a holistic way to simultaneously achieve all three control objectives for systems described by a chain of integrators with input disturbances: 1) global stability of the closed loop system;2) fast overall system response speed for any initial conditions; 3) good disturbance rejection capability at steady state. The overall design philosophy of the proposed holistic approach is outlined below:

- Unlike RG which assumes the use of any inner stabilizing controller and completely ignores the interaction between the design of inner stabilizing controller and the constrained optimization carried out in the outer loop, the proposed approach emphasizes on the seamless integration of the low level trajectory tracking controller and the high level task planning. As such, the proposed approach first seeks for the trajectory tracking controller that explicitly takes into account the input saturation limits to achieve a guaranteed output tracking performance for a class of feasible trajectories even in the presence of disturbances. Specifically, as long as the states of the tracking error dynamics with respect to the replanned trajectory $y_{r}(t)$ are within a pre-specified region $\Omega$ at certain time instance, with the replanned trajectory satisfying certain conditions, the tracking error dynamics will stay inside $\Omega$ thereafter in spite of the disturbances assumed in (3) and exponentially converge to zero when the disturbances disappear, as illustrated in Fig 2. The inner trajectory tracking controller having this property with a guaranteed tracking performance inside $\Omega$ is detailed later and implemented in continuoustime domain.

- In the outer loop, the trajectory $y_{r}(t)$ fed into the inner trajectory tracking controller is replanned such that the initial states of the tracking error dynamics with respect to such a replanned reference trajectory are always within $\Omega$, and the replanned trajectory satisfies all the conditions required by the inner trajectory tracking controller. Specific trajectory replanning algorithms are developed to make $y_{r}(t)$ converges to $y_{d}(t)$ as quickly as possible while satisfying those constraints. This trajectory replanning unit is implemented in discrete-time domain using relatively low sampling rate of $1 / T_{s}$ in order to online compute the optimal reference trajectory which accomplishes the above objectives. Low sampling rate is acceptable here because unlike MPC, the trajectory replanning unit in our case does not have to deal with input disturbances directly.

\section{Problem Formulation}

For simplicity, we consider the following linear system with input disturbances and constraints:

$$
\begin{aligned}
\dot{x}_{1} & =x_{2} \\
& \cdots \\
\dot{x}_{n} & =S(u)+\Delta(t), \\
y & =x_{1}
\end{aligned}
$$

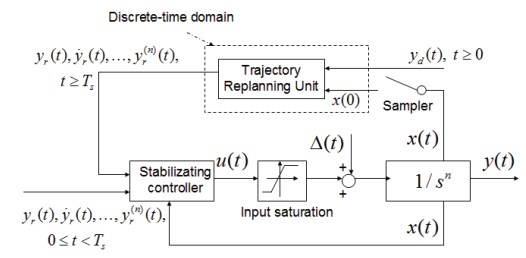

Fig. 1. Closed-loop system structure using the proposed control law

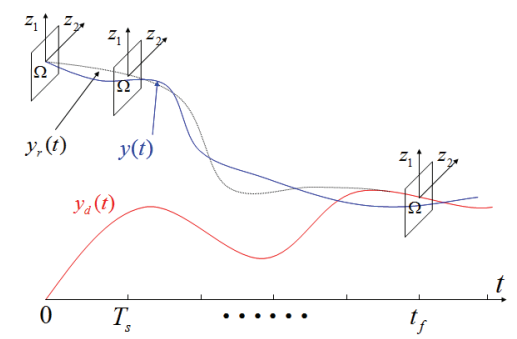

Fig. 2. Illustration of the proposed trajectory replanning approach

where $x=\left[x_{1}, \cdots, x_{n}\right]^{T}$ represents the state vector which is measurable, $y$ is the output, $u$ is the control input to the system, $S(u)$ is the input saturation function given by

$$
S(u)=\left\{\begin{array}{lll}
u, & \text { if } & |u| \leq u_{M} \\
u_{M} \operatorname{sign}(u), & \text { if } & |u|>u_{M},
\end{array}\right.
$$

where $u_{M}$ is the input saturation limit, and $\Delta(t)$ represents the bounded lumped model uncertainties including input disturbances satisfying the following assumption:

Assumption 1: $\Delta(t)$ is bounded within a known value less than the input saturation level, i.e.,

$$
|\Delta(t)| \leq d<u_{M}
$$

Trajectory tracking is considered in this paper. For the desired trajectory to be tracked perfectly in the ideal scenario, it has to satisfy (1) and (2). Thus, it is assumed that the desired trajectory $y_{d}(t)$ is n-th order differentiable and, due to the input saturation limit and the degree of the uncertainties considered in (3), its $n$-th derivative is bounded with a known bound $y_{d M}^{(n)}$ satisfies the following assumption:

Assumption 2:

$$
\sup _{t \geq 0}\left|y_{d}^{(n)}(t)\right|=y_{d M}^{(n)}<u_{M}-d
$$

The objective is to achieve global stability and the output tracking error $e_{y}=y-y_{d}(t)$ converges to zero as fast as possible even in presence of the bounded uncertainties $\Delta$ and the input saturation limit $u_{M}$.

\section{Proposed Controller Design}

\section{A. Design of inner trajectory tracking controller}

Let $\alpha_{0}=y_{r}(t)$, where $y_{r}(t)$ is the replanned trajectory to be synthesized in the next subsection. Define the virtual control 
effort and the tracking error in i-th step $(i<n)$ as

$$
\begin{aligned}
& z_{i}=x_{i}-\alpha_{i-1}\left(z_{1}, \cdots, z_{i-1}, t\right) \\
& \alpha_{i}\left(z_{1}, \cdots, z_{i}, t\right)=y_{r}^{(i)}(t)+ \\
& \sum_{j=1}^{i}\left[(-1)^{i+j-1} \sum_{\substack{m_{1}, \cdots, m_{j} \geq 0 \\
m_{1}+\cdots+m_{j}=i-j+1}}\left(k_{1}^{m_{1}} \cdots k_{j}^{m_{j}}\right) z_{j}\right](6)
\end{aligned}
$$

where $k_{i}>0, \forall i<n$. In the last step ( $\operatorname{step} n$ ), let

$$
\begin{aligned}
z_{n}= & x_{n}-\alpha_{n-1}\left(z_{1}, \cdots, z_{n-1}, t\right) \\
u= & u_{a}+u_{s}, \\
u_{a}= & \sum_{j=1}^{n-1}\left[(-1)^{n+j-1} \sum_{\substack{m_{1}, \cdots, m_{j} \geq 0 \\
m_{1}+\cdots+m_{j}=n-j+1}}\left(k_{1}^{m_{1}} \cdots k_{j}^{m_{j}}\right) z_{j}\right] \\
& -\left(\sum_{j=1}^{n-1} k_{j}\right) z_{n}+y_{r}^{(n)}(t) \\
u_{s}= & -\sigma\left(z_{n}\right) .
\end{aligned}
$$

in which the function $\sigma\left(z_{n}\right)$ is defined as follows:

$\sigma\left(z_{n}\right)= \begin{cases}k_{n} z_{n}, & \text { if }\left|z_{n}\right| \leq l_{n 1} \\ \operatorname{sign}\left(z_{n}\right)\left[k_{n} l_{n 1}+k_{n}^{\prime}\left(\left|z_{n}\right|-l_{n 1}\right)\right], & \text { if } l_{n 1}<\left|z_{n}\right| \leq l_{n} \\ \operatorname{sign}\left(z_{n}\right)\left[k_{n} l_{n 1}+k_{n}^{\prime}\left(l_{n}-l_{n 1}\right)\right], & \text { else }\end{cases}$

where $k_{n}^{\prime} \geq k_{n}$. When $\left|z_{n}\right|=l_{n}, \sigma\left(z_{n}\right)=k_{n} l_{n 1}+k_{n}^{\prime}\left(l_{n}-l_{n 1}\right) \triangleq$ $M$.

With (6) and (5), the error dynamics can be obtained as:

$\dot{z}_{1}=z_{2}-k_{1} z_{1}$

$\cdots$

$\dot{z}_{i}=z_{i+1}-k_{i} z_{i}$

...

$$
\begin{aligned}
\dot{z}_{n}= & S\left(u_{a}+u_{s}\right)+\sum_{j=1}^{n-1}\left[(-1)^{n+j} \sum_{\substack{m_{1}, \cdots, m_{j} \geq 0 \\
m_{1}+\cdots+m_{j}=n-j+1}}\left(k_{1}^{m_{1}} \cdots k_{j}^{m_{j}}\right) z_{j}\right] \\
& +\left(\sum_{j=1}^{n-1} k_{j}\right) z_{n}-y_{r}^{(n)}(t)+\Delta(t)
\end{aligned}
$$

We are interested in finding the positive invariant set of the tracking error $z$. Specifically, once the tracking errors are within this positive invariant set, they will not go out of this set any more. And the control effort needed to confine the error $z$ within the positive invariant set is always within the saturation limits so that no saturation would occur. Such a set is given below:

Theorem 1: With the control law (8), the set $\Omega=\left\{z:\left|z_{i}\right| \leq\right.$ $\left.l_{i}\right\}$ is a positive invariant set and $|u(z, t)|<u_{M}, \forall z \in \Omega$ if the following conditions are satisfied:

$$
\begin{aligned}
& k_{i} l_{i}>l_{i+1}, \quad \forall i<n \\
& M>d \\
& \sum_{j=1}^{n-1}\left[\begin{array}{c}
\left.\sum_{\substack{m_{1}, \cdots, m_{j} \geq 0 \\
m_{1}+\cdots+m_{j}=n-j+1}}\left(k_{1}^{m_{1}} \cdots k_{j}^{m_{j}}\right) l_{j}\right] \\
<u_{M}-\left|y_{r}^{(n)}\right|
\end{array}\right]+\left(\sum_{j=1}^{n-1} k_{j}\right) l_{n}+M \\
& \quad
\end{aligned}
$$

Proof: First, comparing (8)-(11) with the last inequality of (14), we can easily show that $|u(z, t)|<u_{M}, \forall z \in \Omega$. Thus, inside $\Omega$, the dynamics of $z$ can be simplified into:

$$
\begin{array}{rll}
\dot{z}_{1} & =z_{2}-k_{1} z_{1} \\
& \ldots & \\
\dot{z}_{i} & = & z_{i+1}-k_{i} z_{i} \\
& \cdots & \\
\dot{z}_{n} & = & -\sigma\left(z_{n}\right)+\Delta(t)
\end{array}
$$

To prove that $\Omega$ is a positive invariant set is the same as to check that the vector field $\dot{z}$ given by (15) for any $z$ on the boundary of $\Omega$ (denoted as $\partial \Omega$ ) always points inside or along the tangent plane of the boundary. This is shown below.

Suppose that $z=\left[\begin{array}{ll}z_{1}, & z_{2}, \cdots, z_{n}\end{array}\right]^{T} \in \partial \Omega$. Then, some elements $z_{i}$ must hit their boundaries $l_{i}$ or $-l_{i}$ while others are still within their limits. Let $z_{r_{1}}, z_{r_{2}}, \ldots, z_{r_{R}}$ denote the elements that hit their upper bounds, i.e., $z_{r_{j}}=l_{r_{j}}$. Let $z_{p_{1}}$, $z_{p_{2}}, \ldots, z_{p_{P}}$ denote the elements that hit their lower bounds, i.e., $z_{p_{j}}=-l_{p_{j}}$. Let $z_{q_{1}}, z_{q_{2}}, \ldots, z_{q_{Q}}$ denote the elements that are within their limits, i.e., $-l_{q_{j}}<z_{q_{j}}<l_{q_{j}}$. Then it is easy to see that, the vector $\dot{z}$ always points inward if the following conditions are satisfied: $\dot{z}_{r_{j}}<0, \forall j=1, \cdots, R$ and $\dot{z}_{p_{j}}>0$, $\forall j=1, \cdots, P$. These conditions are verified as follows.

$$
\begin{aligned}
& \text { If } r_{j}=n, \dot{z}_{r_{j}}=\dot{z}_{n}=-M+\Delta(t) \leq-M+d<0 . \\
& \text { If } r_{j}=i, i<n, \dot{z}_{r_{j}}=\dot{z}_{i}=z_{i+1}-k_{i} l_{i} \leq l_{i+1}-k_{i} l_{i}<0 . \\
& \text { If } p_{j}=n, \dot{z}_{p_{j}}=\dot{z}_{n}=M+\Delta(t) \geq M-d>0 .
\end{aligned}
$$$$
\text { If } p_{j}=i, i<n, \dot{z}_{p_{j}}=\dot{z}_{i}=z_{i+1}+k_{i} l_{i} \geq-l_{i+1}+k_{i} l_{i}>0 \text {. }
$$

The proof that $\Omega$ is a positive invariant set is now complete.

Theorem 2: Inside $\Omega$, the steady-state output tracking error $z_{1}$ is bounded above by $\left|z_{1}(\infty)\right|<d / \prod_{i=1}^{n} k_{i}$.

Proof: Define $V_{n}=z_{n}^{2} / 2$. From the last inequality of (13), with the condition $k_{n}^{\prime}>k_{n}$, the derivative of $V_{n}$ is given by

$$
\begin{aligned}
& \dot{V}_{n}=z_{n} \dot{z}_{n} \leq\left|z_{n}\right|\left(d-k_{n}\left|z_{n}\right|\right) \leq-\frac{k_{n}}{2} z_{n}^{2}+d\left|z_{n}\right|-\frac{k_{n}}{2} z_{n}^{2} \\
& \leq-\frac{k_{n}}{2}\left(\left|z_{n}\right|-\frac{d}{k_{n}}\right)^{2}+\frac{d^{2}}{2 k_{n}}-\frac{k_{n}}{2} z_{n}^{2} \leq \frac{d^{2}}{2 k_{n}}-\frac{k_{n}}{2} z_{n}^{2} \leq-k_{n} V_{n}+\frac{d^{2}}{2 k_{n}}
\end{aligned}
$$

which leads to $V_{n}(t) \leq e^{-k_{n} t} V_{n}(0)+\frac{d^{2}}{2 k_{n}^{2}}\left[1-e^{-k_{n} t}\right]$. Then, the steady-state value of $z_{n}$ is bounded by $\left|z_{n}(\infty)\right| \leq d / k_{n}$. Noting the first $n-1$ equations of (15), the steady-state output tracking error $z_{1}$ is bounded by $\left|z_{1}(\infty)\right|<d / \prod_{i=1}^{n} k_{i}$, which completes the proof.

Note that within $\Omega$, by choosing $k_{i}$ large enough, the exponential converging rate of all states $z_{i}$ to their steadystate values can be arbitrarily fast and the steady-state output tracking error given by Theorem 2 can be arbitrarily small. The control parameter selection procedure can be as follows:

- Step 1: Choose $k_{1}, k_{2}, \cdots, k_{n}>0$ large enough such that the transient response speed to disturbance is fast enough and the steady-state output tracking performance is met according to Theorem 2 .

- Step 2: Set $M=d+\varepsilon$, where the margin $\varepsilon$ can be any positive value such that $\varepsilon<u_{M}-d-y_{d M}^{(n)}$.

- Step 3: Set $l_{1}=L, l_{2}=\gamma_{1} k_{1} L, \ldots, l_{n}=\prod_{i=1}^{n-1}\left(\gamma_{i} k_{i}\right) L$, where $\gamma_{i}$ can be any value between 0 and 1, i.e., $0<$ $\gamma_{i}<1$. 
- Step 4: Solve for $L$ :

$$
L=\frac{\varepsilon_{1}}{\sum_{j=1}^{n-1}\left[\sum_{\substack{m_{1}, \cdots, m_{j} \geq 0 \\ m_{1}+\cdots+m_{j}=n-j+1}}\left(k_{1}^{m_{1} \ldots k_{j}} m_{j}^{m_{j}}\right) \prod_{r=1}^{j-1}\left(\gamma_{r} k_{r}\right)\right]+\left(\sum_{j=1}^{n-1} k_{j}\right) \prod_{i=1}^{n-1}\left(\gamma_{i} k_{i}\right)}
$$

where $\varepsilon_{1}$ is any value such that $\varepsilon_{1}<u_{M}-d-y_{d M}^{(n)}-$ $\varepsilon$. With this $L$, all $l_{i}$ are determined according to the formula in Step 3.

- Step 5: If $M / l_{n}>k_{n}$, choose $l_{n 1}$ to be any positive value less than $l_{n}$, and $k_{n}^{\prime}=\left(M-k_{n} l_{n 1}\right) /\left(l_{n}-l_{n 1}\right)$. Otherwise reset $l_{n}$ to be $M / k_{n}$, so that $\sigma\left(z_{n}\right)$ becomes a pure linear function inside $\Omega$.

It is trivial to verify that, if $y_{r}^{(n)}$ is chosen such that

$$
\left|y_{r}^{(n)}(t)\right| \leq u_{M}-d-\varepsilon-\varepsilon_{1},
$$

then the above design procedure guarantees that all the inequalities in (14) are satisfied, which provides a clean design interface between the inner-loop tracking controller and the outer-loop trajectory replanning unit.

\section{B. Trajectory Replanning}

After designing the inner stabilizing controller in continuous-time domain, the next step is to synthesize the replanned trajectory $y_{r}(t)$ to prevent saturation and guarantee global stability, and drive the state of the system to the desired trajectory in a finite time.

Let tracking error $z_{d}=\left[z_{1 d}, \cdots, z_{n d}\right]^{T}$ with respect to the desired trajectory be recursively defined as follows:

$$
\begin{aligned}
\alpha_{0} & =y_{d} \\
z_{d i} & =x_{i}-\alpha_{i-1}\left(z_{d 1}, \cdots, z_{d i-1}, t\right) \\
\alpha_{i} & =\sum_{j=1}^{i}\left[(-1)^{i+j-1} \sum_{\substack{m_{1}, \cdots, m_{j} \geq 0 \\
m_{1}+\cdots+m_{j}=i-j+1}}\left(k_{1}^{m_{1}} \cdots k_{j}^{m_{j}}\right) z_{d j}\right]+y_{d}^{(i)}
\end{aligned}
$$

When $z_{d}(0) \in \Omega$, the initial states are close enough to their desired values for perfect tracking of the desired trajectory $y_{d}(t)$. In this case, let the control law $u$ be (8)-(11), where $y_{r}(t)=y_{d}(t)$. Then, (18) is always true and with the design procedure in previous subsection, Theorem 1 guarantees that $z_{d}(t) \in \Omega, \forall t$. So from Theorem 2 , the trajectory tracking will be achieved with a guaranteed accuracy.

When $z_{d}(0) \notin \Omega$, the initial states are too far away from their desired values and the trajectory replanning must be done to prevent control input saturation. Specifically, let the control law be (8)-(11), but with $y_{r}(t)=y_{d}(t)+y_{a}(t)$ where $y_{a}(t)$ is the 'trajectory modification' satisfying the following conditions:

(i) $y_{a}(t)$ is $\mathrm{n}$-th order piecewise continuously differentiable.

(ii) $\left[y_{a}(0), \cdots, y_{a}^{(n-1)}(0)\right]^{T}=\left[x_{1}(0)-y_{d}(0), \cdots, x_{n}(0)-\right.$ $\left.y_{d}^{(n-1)}(0)\right]^{T}$.

(iii) $\left[y_{a}(t), \cdots, y_{a}^{(n-1)}(t)\right]^{T}=0_{n \times 1}, \forall t \geq t_{f}$, where $t_{f}$ is some nonnegative finite value. (iv) $y_{a}^{(n)}(t) \in\left[-u_{M}+d+\varepsilon+\varepsilon_{1}-y_{d}^{(n)}(t)\right.$,

$\left.u_{M}-d-\varepsilon-\varepsilon_{1}-y_{d}^{(n)}(t)\right], \forall t \in\left[0, t_{f}\right]$.

The existence and specific procedure to construct the above trajectory modification will be detailed later.

\section{Main Result (Global Stabilization and Final Tracking Accuracy)}

The following theorem is a direct corollary from the results in the previous sections.

Theorem 3: Consider the inner trajectory tracking control law (8)-(11) with all the controller parameters chosen by Steps 1 to 5 . When the replanned reference trajectory is generated by $y_{r}(t)=y_{d}(t)+y_{a}(t)$ where

- $y_{a}(t)$ satisfies conditions (i)-(iv) if $z_{d}(0) \notin \Omega$,

- $y_{a}(t)=0, \forall t \geq 0$ if $z_{d}(0) \in \Omega$,

then, no matter where the initial states $x(0)$ are, the tracking error $z_{d}$ with respect to the desired trajectory will always enter $\Omega$ in a finite time and will remain in $\Omega$ thereafter. The steady-state output tracking error $z_{d 1}(\infty)$ is bounded above by $\left|z_{d 1}(\infty)\right|<d / \prod_{i=1}^{n} k_{i}$.

\section{Specific trajectory replanning algorithms}

The trajectory modification $y_{a}(t)$ satisfying conditions (i),(iii), and (iv) with its initial conditions chosen according to condition (ii) is not unique. This freedom in choosing $y_{a}(t)$ can be used to meet various objectives in implementation. For example, the freedom can be used to minimize the convergence time $t_{f}$ for fast convergence of the states to the desired trajectory. In this subsection, three specific online trajectory re-planning algorithms will be presented to illustrate how various objectives can be accomplished.

Analytical solution:

The existence of the trajectory modification $y_{a}(t)$ satisfying all the four conditions stated above can be shown via the simple analytical solution given below. Should simplicity of the overall controller be the objective, such an analytical solution can be used as well since it does not need any online optimization algorithm. Specifically, choose $\varepsilon$ and $\varepsilon_{1}$ small enough such that $M_{a}=u_{M}-d-\varepsilon-\varepsilon_{1}-y_{d M}^{(n)}>0$ and analytically generate $y_{a}(t)$ as follows:

Step 1: Let $T_{1}=\frac{\left|y_{a}^{(n-1)}(0)\right|}{M_{a}}$ and $y_{a}^{(n)}(t)=-M_{a} \operatorname{sign}\left(y_{a}^{(n-1)}(0)\right)$, $\forall 0 \leq t<T_{1}$. Analytically generate $y_{a}(t)$ and $y_{a}^{(i)}(t), i=$ $1, \ldots, n-1$ by integration using the initial conditions according to condition (ii). By doing so, $y_{a}^{(n-1)}(t)$ is driven to zero at the end of the time period $\left(0, T_{1}\right]$, i.e., $y_{a}^{(n-1)}\left(T_{1}\right)=0$.

Step i: For $2 \leq i \leq n$, assuming that $y_{a}^{(n-i+j)}(t), j=1, \ldots, i-$ 1 have been driven to zero at the end of the time period $\left(\sum_{j=1}^{i-2} T_{j}, \sum_{j=1}^{i-1} T_{j}\right]$, the step $i$ is to drive $y_{a}^{(n-i+j)}(t), j=1, \ldots, i$ to zero at the end of the time period $\left(\sum_{j=1}^{i-1} T_{j}, \sum_{j=1}^{i} T_{j}\right]$ as follows. Choose $T_{i}=2^{i-1}\left[\frac{\left|y_{a}^{(n-i)}\left(\sum_{j=1}^{i-1} T_{j}\right)\right|}{2^{\frac{(i-2)(i-1)}{2}} M_{a}}\right]^{1 / i}$. Let $y_{a}^{(n)}(t)=$ 
$-M_{a} U(t) \operatorname{sign}\left(y_{a}^{(n-i)}(0)\right), \forall \sum_{j=1}^{i-1} T_{j} \leq t<\sum_{j=1}^{i} T_{j}$, where $U(t)$ is recursively defined as follows:

$$
\begin{aligned}
U(t)= & 1, \forall \sum_{j=1}^{i-1} T_{j} \leq t<\sum_{j=1}^{i-1} T_{j}+\frac{1}{2^{i-1}} T_{i} \\
U(t)= & -1, \forall \sum_{j=1}^{i-1} T_{j}+\frac{1}{2^{i-1}} T_{i} \leq t<\sum_{j=1}^{i-1} T_{j}+\frac{2}{2^{i-1}} T_{i} \\
U(t)= & U\left(t-\frac{\left(2 r+1-2^{f l\left(\log _{2} r\right)+1}\right)}{2^{i-1}} T_{i}\right), \\
& \forall \sum_{j=1}^{i-1} T_{j}+\frac{r}{2^{i-1}} T_{i} \leq t<\sum_{j=1}^{i-1} T_{j}+\frac{r+1}{2^{i-1}} T_{i},
\end{aligned}
$$

in which $f l(a)$ denotes the largest integer less than or equal to $a$ and $r=2, \ldots, 2^{i-1}-1, \forall i>2$. Then, compute $y_{a}(t)$ to $y_{a}^{(n-1)}(t)$ by integrating $y_{a}^{(n)}(t)$ analytically. At time $\sum_{j=1}^{i-1} T_{j}+T_{i}, y_{a}^{(n-1)}, y_{a}^{(n-2)}, \cdots, y_{a}^{(n-i)}=0$.

It is clear that after $n$ steps, at time $t_{f}=\sum_{j=1}^{n} T_{j}$, all the derivatives of $y_{a}(t)$ and $y_{a}(t)$ itself will be zero. Thus, the conditions (i)-(iv) are satisfied with $t_{f}=\sum_{j=1}^{n} T_{j}$.

\section{Numerical time-optimal solution:}

The above analytical solution is obviously not the most timeoptimal one. A general time-optimal solution that satisfies all the conditions can be obtained by solving a time-optimal control problem online. This can be done by adding a trajectory replanning unit in the outer loop which is dedicated to solving the high-level online optimization problem. Suppose that the computation time for solving high-level time-optimal trajectory planning problem is $T_{s}$. Then, if $z_{d}(0) \notin \Omega, y_{a}(t)$ for all $t>0$ is replanned as follows:

1. When $t \in\left[0, T_{s}\right]$, the optimal trajectory is not available yet as it is supposed to take the whole period of $T_{s}$ to execute any trajectory replanning algorithm in the outer loop. During this 'idle period', $y_{a}(t)$ can be arbitrarily specified as long as the condition (i) and (iv) are satisfied. For example, let $y_{a}^{(n)}(t)=0, \forall t \in\left[0, T_{s}\right]$. Then

$$
\begin{aligned}
& y_{a}(t)=y_{a}(0)+\dot{y}_{a}(0) t+\cdots+\frac{1}{(n-1) !} y_{a}^{(n-1)}(0) t^{n-1} \\
& \dot{y}_{a}(t)=\dot{y}_{a}(0)+\ddot{y}_{a}(0) t+\cdots+\frac{1}{(n-2) !} y_{a}^{(n-1)}(0) t^{n-2} \\
& \cdots \\
& y_{a}^{(n-1)}(t)=y_{a}^{(n-1)}(0)
\end{aligned}
$$

At the same time, the high-level trajectory planner is solving a disturbance-free time-optimal control problem by treating $y_{a}^{(n)}(t)$ as the control input, i.e.,

$$
\begin{gathered}
\min _{y_{a}(t), t \in\left[T_{s}, t_{f}\right]} t_{f} \text { subject to } \\
{\left[y_{a}\left(T_{s}\right), \cdots, y_{a}^{(n-1)}\left(T_{s}\right)\right]^{T}=\left[y_{a s 1}, \cdots, y_{a s n}\right]^{T}} \\
{\left[y_{a}\left(t_{f}\right), \cdots, y_{a}^{(n-1)}\left(t_{f}\right)\right]^{T}=0_{n \times 1}} \\
y_{a}^{(n)} \in\left[-u_{M}+d+\varepsilon+\varepsilon_{1}-y_{d}^{(n)}, u_{M}-d-\varepsilon-\varepsilon_{1}-y_{d}^{(n)}\right]
\end{gathered}
$$

where $y_{a s i}$ is obtained by substituting $t=T_{s}$ into each equation of (20) and computing each $y_{a}\left(T_{s}\right)$.

2. When $t \in\left[T_{s}, t_{f}\right]$, in which $t_{f}$ is the optimal end time obtained above, the computed time-optimal $y_{a}(t)$ is fed into the low-level tracking controller as the trajectory modification.

3. When $y>t_{f}, y_{a}(t)=0$.
Note that although this method is implemented in discretetime domain, it only needs to be done for one sampling interval $\left[0, T_{s}\right]$, because the trajectory modification $y_{a}(t)$ for $t \in\left[T_{s}, \infty\right)$ can be completely determined by solving the above time optimal trajectory planning problem only once. It is clearly seen that, $t_{f}$ here is finite because it has to be less than the converging time of the analytical solution presented above which is also finite. Thus, $y_{a}(t)$ generated from the above algorithm strictly satisfy conditions (i)-(iv).

Problem (21) is a disturbance-free time-optimal control problem with input constraint that has been well studied in the past few decades. By applying the maximum principle [17], it can be easily shown that the time-optimal solution $y_{a}^{(n)}(t)$ for problem (21) is a bang-bang control law that has at most $n-1$ switchings between its positive upper limit $u_{M}-$ $d-\varepsilon-\varepsilon_{1}-y_{d}^{(n)}(t)$ and its negative lower limit $-u_{M}+d+\varepsilon+$ $\varepsilon_{1}-y_{d}^{(n)}(t)$ [5]. Thus, a simple numerical algorithm can be worked out for (21) to calculate the switching time series $T_{1}$, $T_{2}, \ldots, T_{n}$ by first expressing $y_{a}^{(m)}(t), m=0, \cdots, n-1$ in terms of $T_{1}, T_{2}, \ldots, T_{n}$ and then solving a set of $n \times n$ equations corresponding to the end condition (condition (iii)). After obtaining the switching time series, $y_{a}^{(m)}(t), m=0, \cdots, n-1$ and $t<t_{f}$ can be analytically expressed as a function of $t$. The detail is omitted here due to the page limit.

Remark 1: The above solution algorithm enforces the constraint (iii) all the time to ensure the control input saturation will never happen, which may not be necessary at the beginning of the run. Intuitively, at the beginning of the run, if the initial states are too far away from the desired trajectory, the most time-optimal control strategy would be to use the maximum available control effort to drive the system state close enough to the desired trajectory first until it has to take action to decelerate to stop. For the numerical optimal solution given above, since a portion of the allowable control effort (i.e., $d+\varepsilon+\varepsilon_{1}$ ) is reserved for disturbance rejection at the steady-state, the overall control input may not be close to the maximum allowable level when the actual magnitude of input disturbances is much smaller than the assumed bound of $d$. In this sense, the solution presented above may still be a bit too cautious overall.

Realizing that the proposed control design always works as long as the states of the tracking error dynamics are within $\Omega$ at some finite time, one can by-pass this conservativeness relatively easily by not enforcing the constraint (iii) at the beginning. Namely, at the beginning, directly use the desired trajectory $y_{d}(t)$ instead of the replanned trajectory $y_{r}(t)$ to calculate the control input $u(z, t)$ given by (8)-(11). If the resulting $u(z, t)$ leads to a saturated control input, then simply use this saturated control input until an unsaturated control input is result. At that time, the proposed on-line trajectory replanning will be used instead.

\section{Simulation}

Consider the following third-order system

$$
\dot{x}_{1}=x_{2}, \dot{x}_{2}=x_{3}, \dot{x}_{3}=S(u)+\Delta, y=x_{1} .
$$


The saturation level is assumed to be $u_{M}=8$. The disturbance term is assumed to be bounded by $d=4$. The desired trajectory is taken to be $y_{d}(t)=\frac{1}{4 \pi^{3}} \sin (2 \pi t)$. Then, $\max \left(y_{d}^{(3)}(t)\right)=2$, which satisfies assumption 2 . The initial states are taken to be $x(0)=\left[\begin{array}{lll}0.5 & 0.2 & 0.5\end{array}\right]^{T}$. The disturbance is $\Delta=2.5+1.5 \sin (2 \pi \times 5 t)$.

In the following methods will be compared:

- Coordinate transformation approach by Teel (C1) [19];

- Low and high gain design (C2) [14], [11];

- Model Predictive Control (C3) [2];

- The approach proposed in this paper with closed-loop poles at -25 for the inner-loop controller and with the numerical trajectory replanning in Section III-D (C4);

- The approach proposed in this paper with the modification in Remark 1 to further speed up the overall system response (C5).

- Reference Governor I with closed-loop poles at -25 (C6): RG approach in [7].

- Reference Governor II with closed-loop poles at -10 (C7);

The sampling rates of $\mathrm{C} 3, \mathrm{C} 6, \mathrm{C} 7$ are taken to be $0.02 \mathrm{sec}$ while the sampling rates of $\mathrm{C} 4$ and $\mathrm{C} 5$ (proposed approaches) are taken to be $0.08 \mathrm{sec}$ to account for potentially heavier computational burden than MPC and RG. The details of the calculations of other controller parameters are omitted due to page limit.

\section{A. Simulation results and discussion}

The output tracking errors and inputs for all controllers are plotted in Fig 3. As shown, due to the inability of $\mathrm{C} 1$ to handle large disturbances, the coordinate transformation approach leads to an unstable closed-loop system. The proposed holistic control strategy $(\mathrm{C} 4, \mathrm{C} 5)$ achieve fastest converging rate as well as the smallest steady-state tracking error. Between them, C5 converges slightly faster than C4 because $\mathrm{C} 5$ uses maximum input voltage $(-8 \mathrm{~V})$ in the first few sampling periods after the idle period. The converging rate of MPC (C3) is almost at the same level as C4 and C5, but the steady-state tracking error is very large due to the poor ability of MPC to handle disturbances. For the low and high gain control law $\mathrm{C} 2$, the steady-state tracking error is acceptable but the response oscillates and converges slowly. For RG approaches, C7 gives good converging speed but poor steady-state tracking accuracy (at the level of $10^{-3}$ ). C6 improves the feedback gain of the inner-loop controller. The steady-state tracking accuracy improves but the converging speed becomes much slower. The reason is that the higher gains used in the inner stabilizing controller make the control input during the inter-sampling periods more prone to input saturation effect, which is completely ignored in the outerloop RG design. This clearly shows that the design of inner stabilizing controller does have significant effect on the achievable performance of the outer loop RG design and the designs of both loops should not be arbitrarily separated.
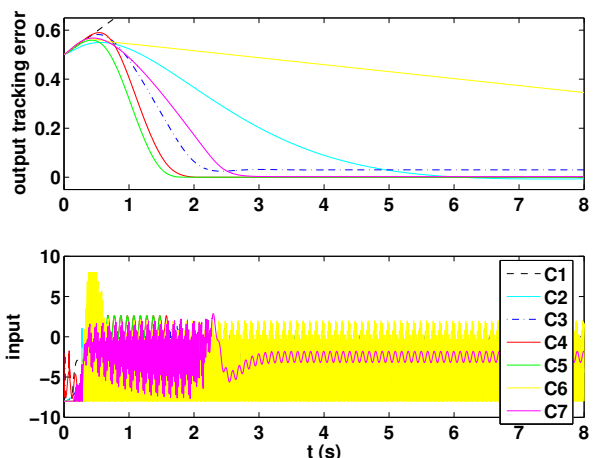

Fig. 3. Output tracking errors and inputs for $\mathrm{C} 1-\mathrm{C} 7$ with disturbance

\section{REFERENCES}

[1] A. Bemporad, "Reference governor for constrained nonlinear systems," IEEE Transactions on Automatic Control, vol. 43, no. 3, pp. 415-419, 1998.

[2] A. Bemporad, M. Morari, and N. L. Ricker, Model predictive control toolbox, user's guide, version 2.1. The MathWorks Inc., 2004.

[3] D. S. Bernstein and A. N. Michel, "A chronological bibliography on saturating actuators," International Journal of Robust and Nonlinear Control, vol. 5, no. 3, pp. 375-380, 1995.

[4] F. Borrelli, Constrained optimal control of linear and hybrid systems. Springer, 2003.

[5] W. D. Collins, "Switching properties of linear time optimal control problems with bounded state constraints," International Journal of Control, vol. 34, no. 1, pp. 111-125, 1981.

[6] E. G. Gilbert and I. Kolmanovsky, "Fast reference governors for systems with states and control constraints and disturbance inputs," International Journal of Robust and Nonlinear Control, vol. 9, no. 15, pp. 1117-1141, 1999.

[7] E. G. Gilbert and C.-J. Ong, "An extended command governor for constrained linear systems with disturbances," in Proceedings of the IEEE Conference on Decision and Control 2009, Shanghai, 2009.

[8] J. Gong and B. Yao, "Global stabilization of a class of uncertain systems with saturatedadaptive robust control," in Decision and Control, 2000. Proceedings of the 39th IEEE Conference on, Sydney, NSW, Australia, December 2000.

[9] G. Grimm, J. Hatfield, I. Postlethwaite, A. R. Teel, M. C. Turner, and L. Zaccarian, "Antiwindup for stable linear systems with input saturation: and lmi-based synthesis," IEEE Transactions on Automatic Control, vol. 48, no. 9, pp. 1509-1525, 2003.

[10] Y. Hong and B. Yao, "A globally stable saturated desired compensation adaptive robust control for linear motor systems with comparative experiments," Automatica, vol. 43, no. 10, pp. 1840-1848, 2007.

[11] T. Hu and Z. Lin, Control System With Actuator Saturation: Analysis and Design. Birkhauser Boston, 2000.

[12] U. Maeder, F. Borrelli, and M. Morari, "Linear offset free model predictive control," Automatica, vol. 45, no. 10, pp. 2214-2222, 2009.

[13] D. Q. Mayne, J. B. Rawlings, C. V. Rao, and P. O. M. Scokaert, "Constrained model predictive control: stability and optimality," $\mathrm{Au}$ tomatica, vol. 36, no. 6, pp. 789-814, 2000.

[14] A. Saberi, Z. Lin, and A. R. Teel, "Control of linear systems with saturating actuators," IEEE Transactions on Automatic Control, vol. 41, no. 41 , pp. 368-378, 1996.

[15] J. Sun and I. Kolmanovsky, "Load governor for fuel cell oxygen starvation protection: a robust nonlinear reference governor approach," IEEE Transactions on Control Systems Technology, vol. 13, no. 6, pp. 911- 920, 2005.

[16] H. J. Sussmann, E. D. Sontag, and Y. Yang, "A general result on the stabilization of linear systems using bounded controls," IEEE Transactions on Automatic Control, vol. 39, pp. 2411-2425, 1994.

[17] H. J. Sussmann and J. C. Willems, "300 years of optimal control: from the brachystochrone to the maximum principle," IEEE Control Systems Magazine, pp. 32-44, June 1997.

[18] A. R. Teel and N. Kapoor, "The 12 anti-windup problem: Its definition and solution," in Proceedings of the 4th ECC, Brussels, Belgium, 1997.

[19] A. Teel, "Global stabilization and restricted tracking for multiple integrators with bounded controls," Systems and Control Letters, vol. 18 , no. 3 , pp. $165-171,1992$. 